\title{
Interaction of low-energy electrons with the pyrimidine bases and nucleosides of DNA
}

\author{
Carl Winstead and Vincent McKoy \\ A. A. Noyes Laboratory of Chemical Physics, California Institute of Technology, Pasadena, \\ California 91125, USA \\ Sergio d'Almeida Sanchez \\ Instituto de Física "Gleb Wataghin," Universidade Estadual de Campinas, 13083-970 Campinas, \\ São Paulo, Brazil
}

(Received 29 May 2007; accepted 19 June 2007; published online 30 August 2007)

\begin{abstract}
We report computed cross sections for the elastic scattering of slow electrons by the pyrimidine bases of DNA, thymine and cytosine, and by the associated nucleosides, deoxythymidine and deoxycytidine. For the isolated bases, we carried out calculations both with and without the inclusion of polarization effects. For the nucleosides, we neglect polarization effects but estimate their influence on resonance positions by comparison with the results for the corresponding bases. Where possible, we compare our results with experiment and previous calculations. () 2007 American Institute of Physics. [DOI: 10.1063/1.2757617]
\end{abstract}

\section{INTRODUCTION}

The surprising observation that slow electrons cause single- and double-strand breaks in DNA (Refs. 1-5) has led to intensive study of the interaction between low-energy electrons and the molecular constituents of DNA and RNA. Because damage is observed at subionization and even subexcitation energies, and because the rate of damage exhibits peaks as a function of the incident electron's kinetic energy, processes involving resonances in the electronically elastic scattering channel are implicated-in particular, resonanceenhanced dissociative attachment (DA). Characterizing the low-energy resonances of nucleic-acid subunits is thus crucial to a full understanding of the mechanism or mechanisms by which slow electrons damage DNA. The considerable experimental attention that has been focused on DA to the nucleobases $^{7-31}$ has not only demonstrated that resonant DA does indeed occur in them but has also led to important mechanistic insights, notably that such resonant DA can be bond selective $\mathrm{e}^{16,23-27}$ and that gas-phase DA may be driven by vibrational Feshbach resonances built on dipole-bound temporary anions, ${ }^{15,23,27}$ rather than on "conventional" elastic shape resonances involving trapping of the electron in an empty valence orbital. Some information is also available concerning DA to the nucleosides deoxythymidine ${ }^{32}$ (dT) and 5-bromouridine. ${ }^{33}$ However, comparatively few studies have directly examined low-energy electron scattering by the nucleobases, nucleosides, or nucleotides. To our knowledge, the only elastic cross section measurement to date is that by Abouaf and Dunet for uracil (and 5-bromouracil), ${ }^{21}$ and their result is relative and limited to $90^{\circ}$ scattering angle. No other differential elastic measurements, and no integral elastic or total scattering cross section measurements, appear to be available. However, electron-transmission studies on the nucleobases by Gallup, Burrow, and co-workers ${ }^{15,34}$ have revealed the energies and widths of low-energy resonances, which they have assigned as valence shape resonances asso- ciated with the vacant $\pi^{*}$ orbitals. Cross section calculations have generally relied on one-electron models ${ }^{35-38}$ and have not yielded resonance positions in good agreement with experiment. ${ }^{15,34,36,38,39}$

In three recent papers, we have reported electron collision cross sections computed from first principles for various nucleic-acid constituents, including the RNA base uracil, ${ }^{40}$ the purine bases and nucleosides together with the purine nucleotide deoxyadenosine $5^{\prime}$-monophosphate, ${ }^{41}$ and the backbone sugar deoxyribose as well as its $5^{\prime}$ phosphate ester. ${ }^{42}$ Our all-electron results for the $\pi^{*}$ resonances in uracil (U) and the purine bases were in better, though far from perfect, agreement with the measured resonance positions than previous model-potential results. In the present paper, we consider elastic electron collisions with the pyrimidine bases of DNA, thymine (T) and cytosine (C), and with the corresponding nucleosides, $\mathrm{dT}$ and deoxycytidine (dC). Structures for these molecules are shown in Fig. 1. As in previous work, ${ }^{40,41}$ we focus on the energies of the $\pi^{*}$ shape resonances, and we use comparison between lowerand higher-level results in the isolated bases, along with comparison between calculation and experiment, to estimate the actual resonance positions in the nucleosides.

The next section gives details of the calculations. Section III contains the results and discussion, and Sec. IV summarizes our results.

\section{COMPUTATIONAL DETAILS}

The Schwinger multichannel method ${ }^{43,44}$ and its implementation ${ }^{45,46}$ have been described elsewhere. Here we give only the particulars of the present calculations.

We optimized the ground-state nuclear geometries of $\mathrm{C}$ and $\mathrm{T}$ at the level of second-order Möller-Plesset perturbation theory (MP2) within the 6-31G $(d)$ basis set, using either GAMESS (Ref. 47) or GAUSSIAN $94,{ }^{48}$ with the constraint of $C_{s}$ 

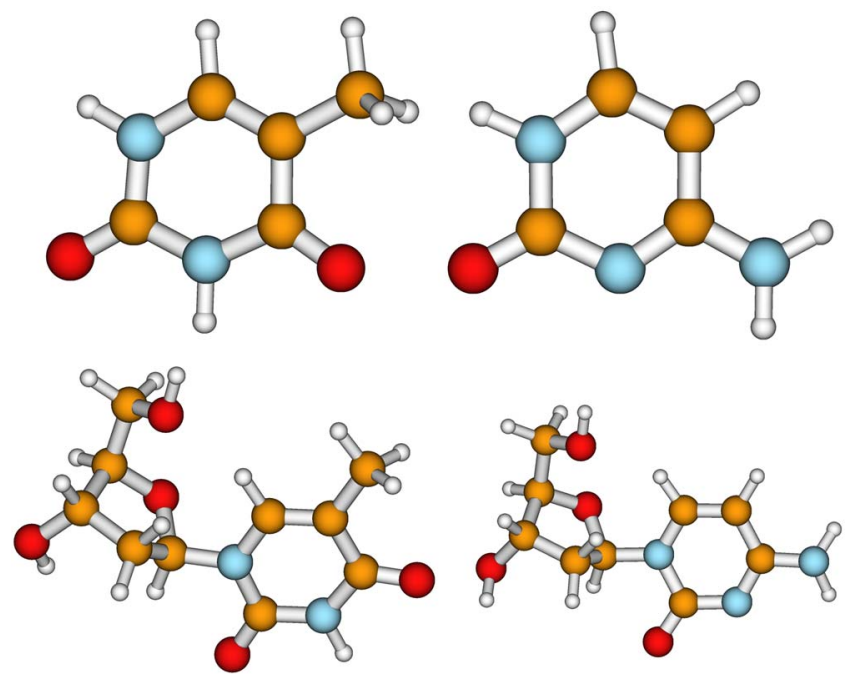

FIG. 1. (Color online) The molecules considered in the present work. On the top row are the pyrimidine bases thymine (left) and cytosine (right). Below each base is shown the corresponding $2^{\prime}$-deoxy nucleoside. Oxygen is red (dark), carbon brown (medium), nitrogen blue (light), and hydrogen white.

point-group symmetry imposed. The $C_{S}$ constraint facilitates the scattering calculations, but forcing a planar geometry on the amino group of $\mathrm{C}$ may slightly shift resonance positions. Trial calculations on $\mathrm{C}$ in a small basis set indicate that the shifts are no more than $\sim 0.2 \mathrm{eV}$, comparable to those we saw for guanine. ${ }^{41}$ For dT, we optimized the geometry at the MP2/6-31+G(d) level, that is, with the 6-31G(d) basis set supplemented by a $1 s 1 p$ set of diffuse Gaussians (for which we used the GAMESS default values) on the heavy atoms. Our MP2/6-31+ $\mathrm{G}(d)$ geometry for $\mathrm{dT}$ is almost identical to that which we obtain at the MP2/6-31G $(d)$ level, which is in turn very similar to the MP2/6-31G $(d)$ geometry determined by Foloppe and MacKerell ${ }^{49}$ for the "south" conformer of the furanose ring. For dC, we used the "south" MP2/6-31G(d) geometry of Foloppe and MacKerell. ${ }^{49}$ The structures of the four molecules are shown in Fig. 1, which was generated using MOLDEN. ${ }^{50}$

For $\mathrm{C}$ and $\mathrm{T}$, we carried out both static-exchange (SE) and static-exchange plus polarization (SEP) calculations of the elastic electron-scattering cross sections. Final SE results for $\mathrm{C}$ were obtained in a large basis set intended to capture some of the effects of long-range collisions mediated by the dipole potential. This set comprised the "triple zeta valence" (TZV) basis of Dunning ${ }^{51}$ together with a $3 d$ set of polarization functions (from which the $x^{2}+y^{2}+z^{2}$ combination was excluded), a $2 p$ polarization set on the hydrogens, a diffuse supplement at the positive end of the molecule, and a set of $s$-type Gaussians distributed on a cubic grid. GAMESS default exponents and splitting factors were used for the polarization functions, while the diffuse supplement and $s$ grid were the same as previously used for $\mathrm{U}^{40}$ with the center for the diffuse functions shifted to an appropriate location for C. This basis contained 803 contracted Gaussian functions. Final SE results for $\mathrm{T}$ were obtained in a smaller basis set, consisting of the "double zeta valence" (DZV) of Dunning and Hay ${ }^{52}$ together with a standard supplement of diffuse and polarization functions: $1 s 1 p 2 d$ on the heavy atoms and $1 s 1 p$ on the hydrogens, again using the default exponents and $d$ splitting factor of GAMESS. ${ }^{47}$

For the $A^{\prime \prime}$ symmetry of $\mathrm{C}$ and $\mathrm{T}$, we carried out SEP calculations in order to obtain more accurate $\pi^{*}$ resonance energies. For $\mathrm{C}$, we performed SEP calculations in the same three basis sets as used in earlier work on $\mathrm{U}{ }^{40}$ The smallest, basis $\mathrm{A}$, is the $6-311 \mathrm{G}++(d, p)$ basis set as defined in GAMESS ${ }^{47}$ with default exponents for the diffuse and polarization functions and with the $x^{2}+y^{2}+z^{2}$ combination of $d$ functions excluded. Basis B is formed by supplementing basis A with diffuse $s$ and $p$ Gaussians located both at the ring center and at the positive end of the molecule. Basis C consists of the $\operatorname{TZV}(2 d, p)$ basis set of GAMESS, with default exponents and splitting factors for the polarization functions and the " $3 s$ " combination of $d$ orbitals excluded, together with distributed $s$ Gaussians and a set of diffuse functions at the positive end of the molecule. The centers on which supplementary functions were placed were shifted appropriately to take into account the differences in structure and dipole moment direction between cytosine and uracil. For T, we performed $A^{\prime \prime}$ SEP calculations in bases $\mathrm{A}$ and $\mathrm{B}$.

The closed-channel configuration state functions (CSFs) included in the $A^{\prime \prime}$ SEP calculations were chosen to describe relaxation of the target molecule's electron density in the presence of an electron trapped in one of the $\pi^{*}$ orbitals. An electron was placed in any of the three lowest-energy $a^{\prime \prime}$ modified virtual orbitals ${ }^{53}$ formed for a +6 cationic Fock operator, and all symmetry-preserving singlet-coupled single excitations from the valence occupied orbitals into any of the virtual orbitals were allowed. This procedure results in configuration spaces of manageable size $\left(\sim 10^{4} \mathrm{CSFs}\right)$ and has proven effective in capturing resonant polarization in past work, ${ }^{54}$ but its success depends, of course, on whether the underlying assumption that orbital relaxation is the only significant polarization effect is correct. We will return to this point in Sec. IV.

For the larger molecules, dC and dT, we carried out only SE calculations, for which we used the same DZV plus $1 s 1 p 2 d$ basis set as in the SE calculations on T, henceforth referred to as basis D. In a prior study of purine bases and nucleosides, ${ }^{41}$ we found that this basis set gave wellconverged SE cross sections in the energy range where the $\pi^{*}$ resonances occur, although it does not capture dipolarscattering effects that are important at the very lowest energies.

The forward differential cross sections will be strongly influenced by the long-range interaction between the projectile electron and the static electric dipole moments of the molecules considered here, an effect that is not fully captured in our calculations (though the SE calculations in large basis sets do capture it partially). Procedures exist ${ }^{55}$ for correcting calculated scattering amplitudes to account for such longrange interactions; however, they are not expected to affect the $\pi^{*}$ resonance energies significantly, and we have omitted such corrections in obtaining the present results. 


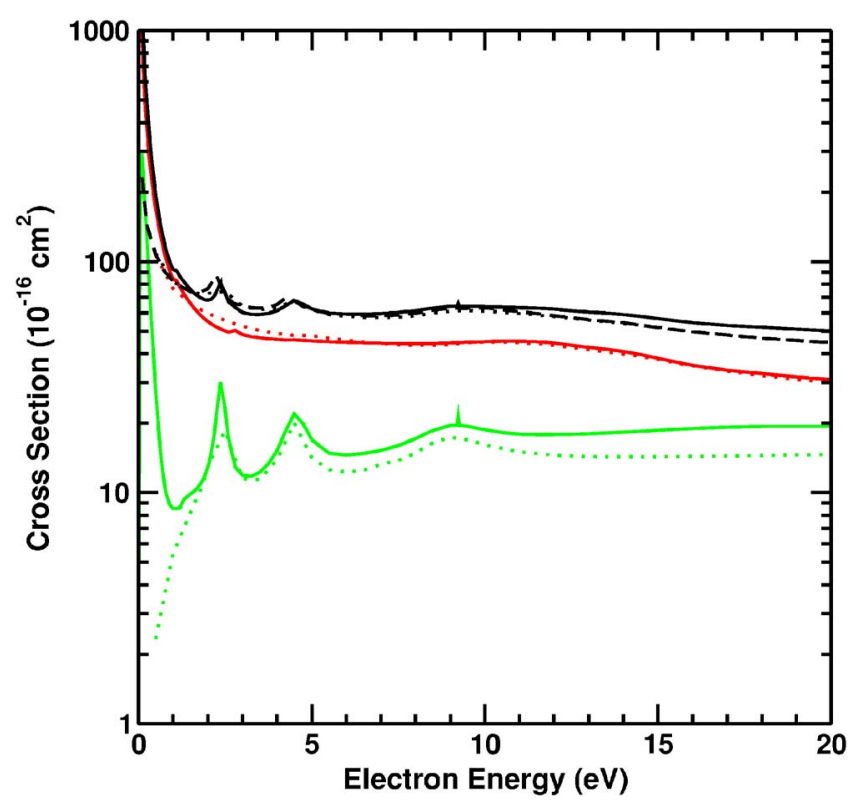

FIG. 2. (Color online) Elastic cross sections for cytosine computed in the static-exchange approximation. The solid curves are the final results obtained in the large basis set and the dotted curves the results obtained in a small basis, basis A (see text for discussion). The dashed curve is obtained in basis A without the imposition of $C_{s}$ symmetry. The top three curves (black) are the integral cross sections; the middle two curves (red) are the $A^{\prime}$ symmetry component; and the bottom two curves (green) are the $A^{\prime \prime}$ component, in which the $\pi^{*}$ resonances occur.

\section{RESULTS AND DISCUSSION}

\section{A. Cytosine}

In Fig. 2, we show the integral cross section (ICS) for electron scattering by $\mathrm{C}$ obtained within the static-exchange approximation. The solid curves show the final results for the ICS and its $C_{s}$ symmetry components obtained in the extended basis set described in the preceding section. For comparison, the dotted curves show $C_{s}$ results obtained in the smaller basis A, while the dashed curve is the ICS obtained in basis A without the imposition of $C_{s}$ symmetry. Clearly, the two $C_{s}$ calculations agree closely on the positions of the three $\pi^{*}$ resonances, while the $C_{1}$ calculation indicates only a slight shift toward lower energy. The main differences between the results obtained in the larger and the smaller basis sets occur at very low energy, where the extended basis does a better job of representing the enhancement of the cross section by dipolar scattering, and at energies above the second $\pi^{*}$ resonance, where the larger basis set produces a somewhat larger cross section.

SEP results for the $A^{\prime \prime}$ component of the ICS are shown in Fig. 3. As was the case for $\mathrm{U}^{40}$ the $\pi^{*}$ resonances of $\mathrm{C}$ appear to be mixed with pseudoresonances, and our usual procedure $^{56}$ for dealing with the latter is only partially successful, particularly in the vicinity of the lowest-energy $\pi^{*}$ resonance, which sits atop a strong dipolar-scattering background. To illustrate these points, Fig. 3 includes both the "raw" results including pseudoresonances (upper panel) and the results after removing a single ${ }^{2} A$ " vector (associated with the smallest singular value of the matrix we are inverting) from each calculation (lower panel). Three features clearly persist in the latter case, and we associate these with

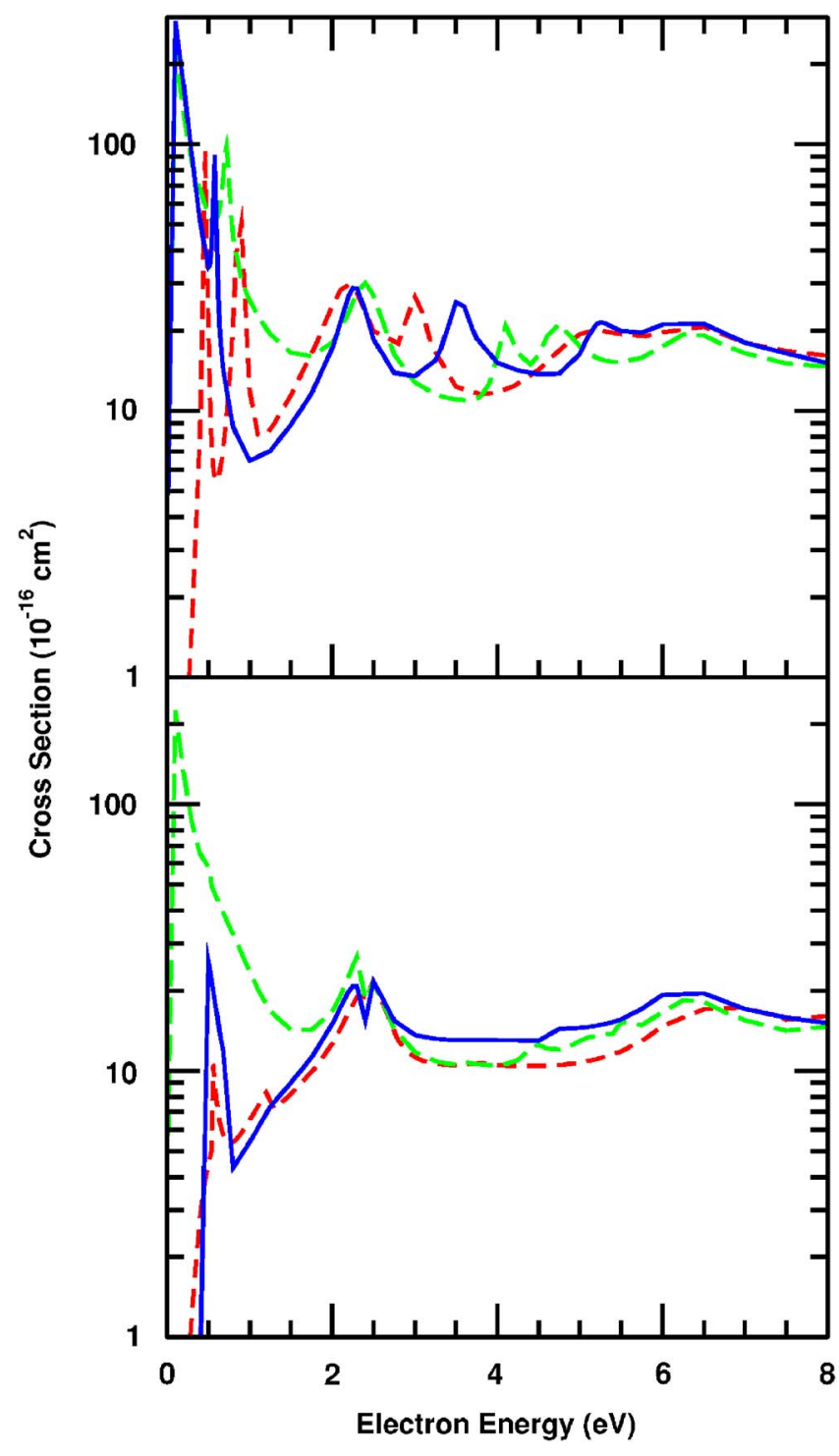

FIG. 3. (Color online) $A^{\prime \prime}$ component of the low-energy integral cross section for elastic scattering of electrons by cytosine computed in the SEP approximation. The short red dashes are results from basis $\mathrm{A}$, the long green dashes from basis B, and the solid blue lines from basis C. The top panel shows results with no attempt to control numerical singularity and the bottom panel results with one vector removed from each calculation.

the $\pi^{*}$ resonances. However, the lowest-energy feature appears only as a shoulder in basis B, while its shape and width are probably distorted in bases A and C. On the other hand, there is quite good agreement among the three basis sets for the second $\pi^{*}$ resonance, while the basis A result places the third resonance somewhat higher than do the two larger calculations. The narrow "window" in the center of the second resonance is a numerical artifact produced by our procedure for dealing with near singularity in our linear equations. We are currently investigating refined numerical procedures that will remove more completely structure due to near singularity or linear dependence while avoiding such artifacts.

The $\pi^{*}$ resonance positions obtained from the SEP calculations, Fig. 3, show the expected downward shift in energy compared to the SE results of Fig. 2. Our SEP resonance energies of $0.5,2.4$, and $6.3 \mathrm{eV}$ are somewhat higher than the positions measured in electron transmission, ${ }^{34}$ 


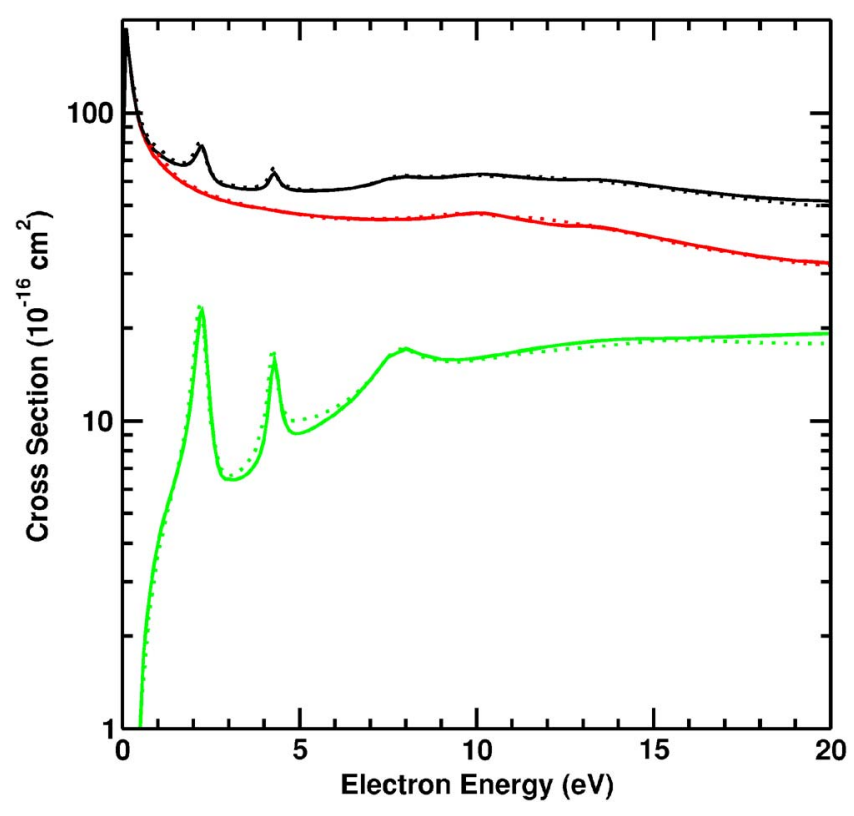

FIG. 4. (Color online) Elastic cross sections for thymine computed in the static-exchange approximation. The solid curves are the final results obtained in basis D and the dotted curves the results obtained in basis A (see text for discussion). The top curves (black) are the integral cross sections; the middle curves (red) are the $A^{\prime}$ symmetry component; and the bottom curves (green) are the $A^{\prime \prime}$ component, in which the $\pi^{*}$ resonances occur.

namely, $0.32,1.53$, and $4.50 \mathrm{eV}$, indicating that our treatment of polarization is not completely adequate. Previous calculations by Tonzani and Greene ${ }^{38}$ using a local-potential model for both exchange and polarization predicted $\pi^{*}$ energies of $1.7,4.3$, and $8.1 \mathrm{eV}$, considerably above the experimental resonance positions. Because the model of Tonzani and Greene gives a SE energy about $2 \mathrm{eV}$ too high for the ${ }^{2} \Pi_{u}$ shape resonance in $\mathrm{CO}_{2},{ }^{57}$ it appears likely that errors due to the treatment of exchange in Ref. 38 are partially offset by inclusion of an attractive polarization potential, yielding resonance positions that are close to our SE results. Similar behavior was found for U (Ref. 40) and the purine bases. $^{41}$

\section{B. Thymine}

Our SE results for the ICS of T are presented in Fig. 4. The results obtained in bases A and D are very similar. Because neither basis set includes an extensive supplement of diffuse and distributed Gaussians, the cross section does not show as much enhancement at very low energies as we saw in the large-basis results for C. However, as seen in Fig. 2, the behavior of the cross section at higher energies, including the $\pi^{*}$ resonance positions, was scarcely affected by use of an extended basis set.

SEP results for $\mathrm{T}$ are shown in Fig. 5. Once again, as for $\mathrm{U}$ (Ref. 40) and C (Fig. 3), the cross section displays both the expected $\pi^{*}$ resonances and additional peaks. From consideration of the effect of deleting the ${ }^{2} A$ " vector associated with the smallest singular value of the linear system, comparison between the two basis sets, and comparison with the corresponding SEP results for $\mathrm{C}$ and $\mathrm{U}$, we can identify the peaks at $0.3,1.9$, and $5.7 \mathrm{eV}$ as the $\pi^{*}$ resonances. For the first two resonances, these SEP energies agree quite well

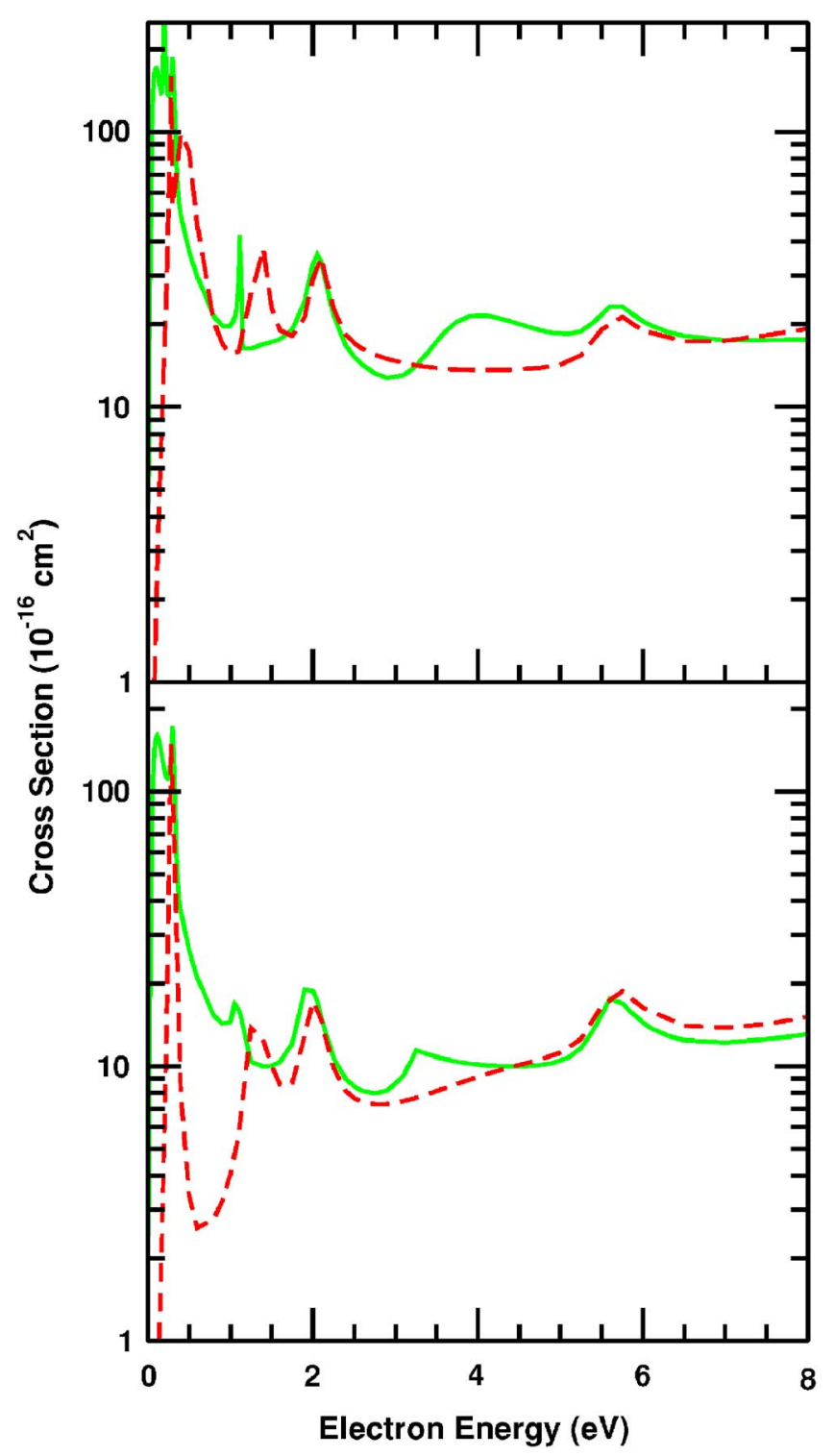

FIG. 5. (Color online) $A^{\prime \prime}$ component of the low-energy integral cross section for elastic scattering of electrons by thymine computed in the SEP approximation. The short red dashes are results from basis A and the solid green lines results from basis B. The top panel shows results with no attempt to control numerical singularity and the bottom panel results with one vector removed from each calculation.

with the measured values of 0.29 and $1.71 \mathrm{eV},{ }^{34}$ but the third resonance is much further above its measured position, $4.05 \mathrm{eV}$. The peaks near $1.0 \mathrm{eV}$ and between 3 and $4 \mathrm{eV}$ in basis B appear to be artifactual, and there are no corresponding features in the transmission measurement. The calculation of Tonzani and Greene ${ }^{38}$ places the $\pi^{*}$ resonances in T at 2.4, 5.5, and $7.9 \mathrm{eV}$.

\section{Larger moieties}

In Figs. 6 and 7, we show the SE results for the nucleosides $\mathrm{dC}$ and $\mathrm{dT}$, respectively, along with $\mathrm{SE}$ results for the corresponding pyrimidine bases, $\mathrm{C}$ and $\mathrm{T}$. All of the results in Figs. 6 and 7 are obtained in basis D except those for C, for which basis $\mathrm{C}$ was employed. Other than an overall increase in magnitude, the nucleoside cross sections show a strong resemblance to those of the corresponding bases. However, 


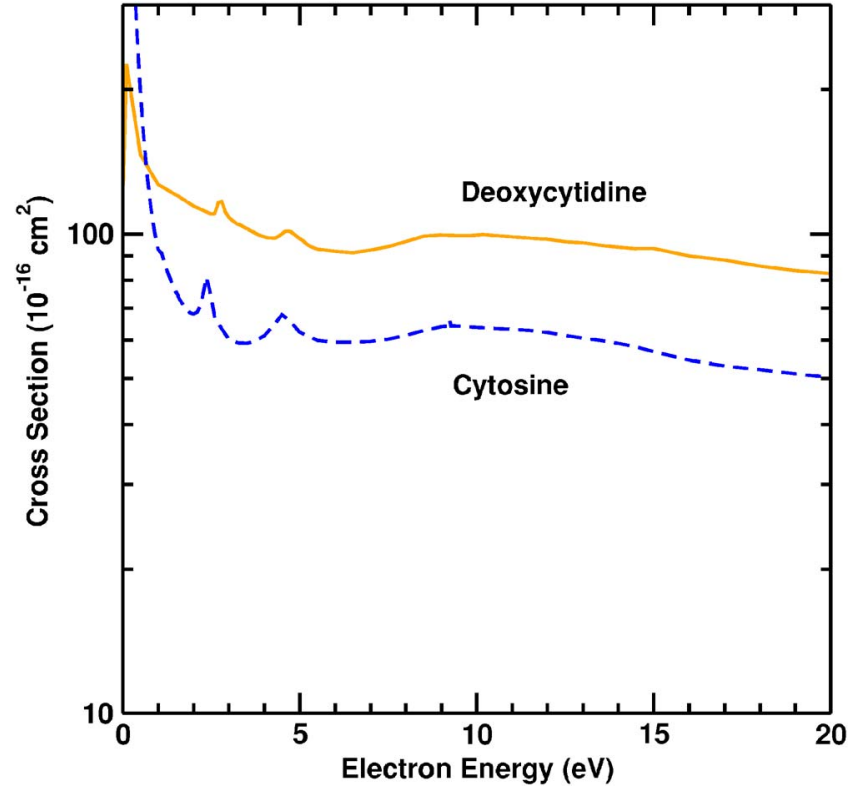

FIG. 6. (Color online) Integral elastic cross section for electron scattering by $2^{\prime}$-deoxycytidine (solid orange line), computed in the static-exchange approximation. The static-exchange cross section for cytosine is shown for comparison (dashed blue line).

on close inspection, one may observe a slight upward shift of the two lowest $\pi^{*}$ resonances both for $\mathrm{dC}$ relative to $\mathrm{C}$ and for $\mathrm{dT}$ relative to $\mathrm{T}$. We observed comparable destabilization by $\sim 0.1-0.3 \mathrm{eV}$ of the low-lying $\pi^{*}$ resonances in our study of the purine nucleosides. ${ }^{41}$ A reasonable first estimate for the actual resonance positions in the nucleosides may thus be obtained by applying comparable upward shifts to the observed resonance positions ${ }^{34}$ for $\mathrm{C}$ and $\mathrm{T}$.

Abdoul-Carime et al. $^{32}$ studied dissociative attachment to gas-phase dT. The principal anions they observed were

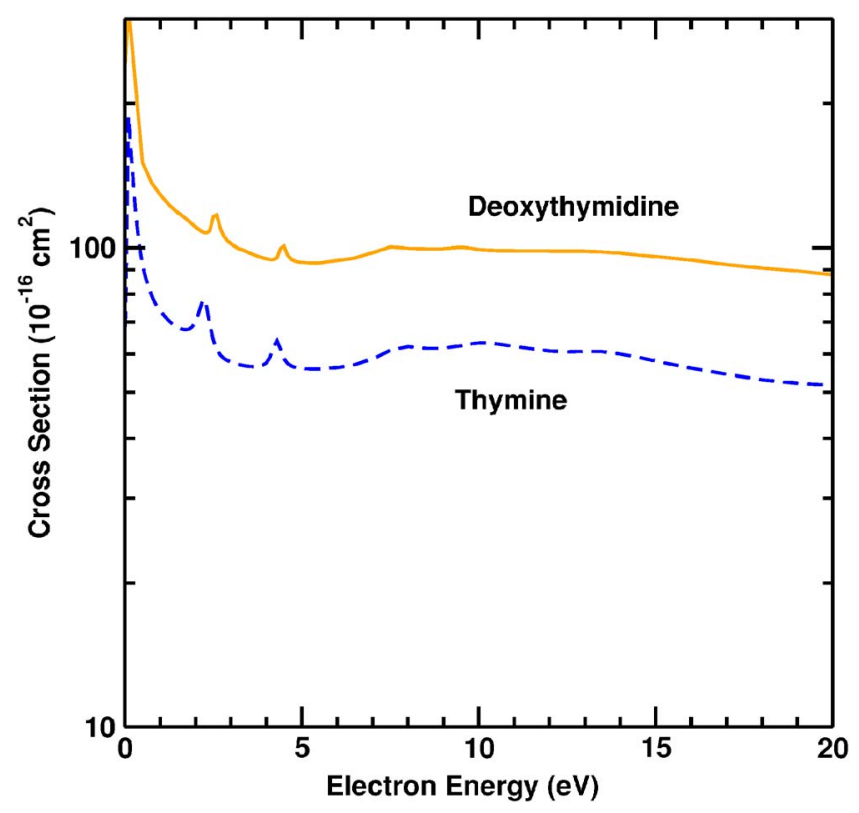

FIG. 7. (Color online) Integral elastic cross section for electron scattering by $2^{\prime}$-deoxythymidine (solid orange line), computed in the static-exchange approximation. The static-exchange cross section for thymine is shown for comparison (dashed blue line).
$(\mathrm{T}-\mathrm{H})^{-}$, that is, the anion of a dehydrogenated thyminyl radical, and (2-deoxyribose- $\mathrm{OH})^{-}$. Each of these anions could arise from electron attachment to dT followed by cleavage of the $\mathrm{N}_{1}$-glycosidic (base-sugar) bond. The yield curves for both of these anions show weak maxima near $0.3 \mathrm{eV}$. At higher energy, the $(\mathrm{T}-\mathrm{H})^{-}$yield closely resembles that observed in DA measurements on thymine itself, 7,11,12,16,17,21,25-27,29 with a narrow peak near $1.0 \mathrm{eV}$ and a broader maximum centered at about $1.7 \mathrm{eV}$, while the (2-deoxyribose- $\mathrm{OH})^{-}$yield shows a single broad maximum centered near $1.2 \mathrm{eV}$. Since our calculations indicate only small shifts in the resonance positions between $\mathrm{T}$ and $\mathrm{dT}$, the peak at $1.7 \mathrm{eV}$ in $(\mathrm{T}-\mathrm{H})^{-}$yield from $\mathrm{dT}$ might be associated with the second $\pi^{*}$ resonance, as has been suggested for the analogous peak in DA to $\mathrm{T}^{27}$ Likewise, the weak feature at $0.3 \mathrm{eV}$ might be associated with trapping in the first $\pi^{*}$ resonance. On the other hand, given the destabilization of the nucleoside $\pi^{*}$ resonances relative to the nucleobase resonances that is indicated by our calculations, we would have anticipated some shift in the DA peak positions between $\mathrm{T}$ and dT. Moreover, the strong peak at $1.0 \mathrm{eV}$, which does not appear to correlate with any shape resonance, is puzzling. This peak is entirely suppressed in DA to $\mathrm{N}_{1}$-methyl thymine, ${ }^{26,27}$ consistent with the hypothesis ${ }^{15,23,27}$ that it is due to a vibrational Feshbach resonance built on the dipolebound anion of T. Attachment of a sugar rather than a methyl at $\mathrm{N}_{1}$ should be even more effective at preventing the formation and subsequent dissociation of a dipole-bound state. Accordingly, Burrow et al. ${ }^{27}$ have suggested that the $(\mathrm{T}-\mathrm{H})^{-}$ anions observed by Abdoul-Carime et al. ${ }^{32}$ arise not from DA to dT itself but from DA to $\mathrm{T}$ produced by inadvertent thermolysis of dT. Further investigation of electron interactions with the nucleosides is clearly warranted.

\section{SUMMARY}

We have reported cross sections for elastic electron scattering by cytosine, thymine, 2 '-deoxycytidine, and $2^{\prime}$-deoxythymidine. Our best (SEP) values for the energies of the $\pi^{*}$ shape resonances in $\mathrm{C}$ and $\mathrm{T}$ are reasonably close to, and therefore supportive of, the positions that have been assigned based on electron transmission measurements, and lower than those obtained in previous calculations. The disagreement between our SEP results and experiment is largest for the third $\pi^{*}$ resonance, indicating that our description of polarization effects in the case of this resonance is less satisfactory than it is for the two lower-energy resonances. The explanation for this discrepancy appears to lie in the mixing of the third resonance with core-excited resonances built on one or more of the low-lying triplet states, as we recently described elsewhere. ${ }^{58}$ In future work on the DNA and RNA bases, we hope to account more completely for such channel mixing.

The cross sections for the pyrimidine nucleosides exhibit the same pattern that we previously observed ${ }^{41}$ for the purines: a strong qualitative similarity to the cross section for the corresponding nucleobase, but with a slight destabilization of the low-energy $\pi^{*}$ resonances. We did not observe any further shift in resonance positions on going from the 
nucleoside to the $5^{\prime}$ nucleotide in the case of deoxyadenosine monophosphate, and it is therefore likely that there will be little if any such shift in the pyrimidine case. These results are thus a first step toward estimating resonance positions in DNA itself, though effects of solvation, base pairing, and base stacking, which we have not yet considered, may prove significant.

\section{ACKNOWLEDGMENTS}

We gratefully acknowledge support of this work by the U.S. Department of Energy, Office of Basic Energy Sciences, and use of the computational resources of the Caltech-JPL Supercomputing Project. One of the (S.d'A.S.) acknowledges support by the Brazilian agencies CAPES and FAPESP.

${ }^{1}$ B. Boudaïfa, P. Cloutier, D. Hunting, M. A. Huels, and L. Sanche, Science 287, 1658 (2000).

${ }^{2}$ B. Boudaïfa, P. Cloutier, D. Hunting, M. A. Huels, and L. Sanche, Radiat. Res. 157, 227 (2002)

${ }^{3}$ M. A. Huels, B. Boudaïfa, P. Cloutier, D. Hunting, and L. Sanche, J. Am. Chem. Soc. 125, 4467 (2003).

${ }^{4}$ F. Martin, P. D. Burrow, Z. Cai, P. Cloutier, D. Hunting, and L. Sanche, Phys. Rev. Lett. 93, 068101 (2004).

${ }^{5}$ R. Panajotovic, F. Martin, P. Cloutier, and L. Sanche, Radiat. Res. 165, 452 (2006).

${ }^{6}$ For a recent review, see L. Sanche, Eur. Phys. J. D 35, 367 (2005).

${ }^{7}$ M. A. Huels, I. Hahndorf, E. Illenberger, and L. Sanche, J. Chem. Phys. 108, 1309 (1998)

${ }^{8}$ H. Abdoul-Carime, M. A. Huels, E. Illenberger, and L. Sanche, J. Am. Chem. Soc. 123, 5354 (2001).

${ }^{9}$ M.-A. Hervé du Penhoat, M. A. Huels, P. Cloutier, J.-P. Jay-Gerin, and L. Sanche, J. Chem. Phys. 114, 5755 (2001).

${ }^{10}$ H. Abdoul-Carime, P. Cloutier, and L. Sanche, Radiat. Res. 155, 625 (2001).

${ }^{11}$ R. Abouaf, J. Pommier, and H. Dunet, Int. J. Mass Spectrom. 226, 397 (2003).

${ }^{12}$ S. Denifl, S. Ptasinska, M. Cingel, S. Matejcik, P. Scheier, and T. D. Märk, Chem. Phys. Lett. 377, 74 (2003).

${ }^{13}$ S. Gohlke, H. Abdoul-Carime, and E. Illenberger, Chem. Phys. Lett. 380, 595 (2003).

${ }^{14}$ G. Hanel, B. Gstir, S. Denifl, P. Scheier, M. Probst, B. Farizon, M. Farizon, E. Illenberger, and T. D. Märk, Phys. Rev. Lett. 90, 188104 (2003)

${ }^{15}$ A. M. Scheer, K. Aflatooni, G. A. Gallup, and P. D. Burrow, Phys. Rev. Lett. 92, 068102 (2004).

${ }^{16}$ H. Abdoul-Carime, S. Gohlke, and E. Illenberger, Phys. Rev. Lett. 92, 168103 (2004).

${ }^{17}$ S. Denifl, S. Ptasińska, M. Probst, J. Hrušak, P. Scheier, and T. D. Märk, J. Phys. Chem. A 108, 6562 (2004).

${ }^{18}$ S. Denifl, S. Ptasińska, G. Hanel, B. Gstir, M. Probst, P. Scheier, and T. D. Märk, J. Chem. Phys. 120, 6557 (2004).

${ }^{19}$ S. Feil, K. Gluch, S. Matt-Leubner, P. Scheier, J. Limtrakul, M. Probst, H. Deutsch, K. Becker, A. Stamatovic, and T. D. Märk, J. Phys. B 37, 3013 (2004)

${ }^{20}$ H. Abdoul-Carime, J. Langer, M. A. Huels, and E. Illenberger, Eur. Phys.
J. D 35, 399 (2005).

${ }^{21}$ R. Abouaf and H. Dunet, Eur. Phys. J. D 35, 405 (2005).

${ }^{22}$ K. Aflatooni, A. M. Scheer, and P. D. Burrow, Chem. Phys. Lett. 408, 426 (2005).

${ }^{23}$ A. M. Scheer, C. Silvernail, J. A. Belot, K. Aflatooni, G. A. Gallup, and P. D. Burrow, Chem. Phys. Lett. 411, 46 (2005).

${ }^{24}$ S. Ptasinska, S. Denifl, V. Grill, T. D. Märk, P. Scheier, S. Gohlke, M. A. Huels, and E. Illenberger, Angew. Chem., Int. Ed. 44, 1647 (2005).

${ }^{25}$ S. Ptasińska, S. Denifl, B. Mróz, M. Probst, V. Grill, E. Illenberger, P. Scheier, and T. D. Märk, J. Chem. Phys. 123, 124302 (2005).

${ }^{26}$ S. Ptasinska, S. Denifl, P. Scheier, E. Illenberger, and T. D. Märk, Angew. Chem., Int. Ed. 44, 6941 (2005).

${ }^{27}$ P. D. Burrow, G. A. Gallup, A. M. Scheer, S. Denifl, S. Ptasinska, T. Märk, and P. Scheier, J. Chem. Phys. 124, 124310 (2006).

${ }^{28}$ S. Denifl, F. Zappa, I. Mähr, J. Lecointre, M. Probst, T. D. Märk, and P. Scheier, Phys. Rev. Lett. 97, 043201 (2006).

${ }^{29}$ K. Aflatooni, A. M. Scheer, and P. D. Burrow, J. Chem. Phys. 125, 054301 (2006).

${ }^{30}$ D. Huber, M. Beikircher, S. Denifl, F. Zappa, S. Matejcik, A. Bacher, V. Grill, T. D. Märk, and P. Scheier, J. Chem. Phys. 125, 084304 (2006).

${ }^{31}$ I. I. Shafranyosh, M. I. Sukhoviya, and M. I. Shafranyosh, J. Phys. B 39, 4155 (2006)

${ }^{32}$ H. Abdoul-Carime, S. Gohlke, E. Fischbach, J. Scheike, and E. Illenberger, Chem. Phys. Lett. 387, 267 (2004).

${ }^{33}$ S. Denifl, P. Candori, S. Ptasińska, P. Limão-Vieira, V. Grill, T. D. Märk, and P. Scheier, Eur. Phys. J. D 35, 391 (2005).

${ }^{34}$ K. Aflatooni, G. A. Gallup, and P. D. Burrow, J. Phys. Chem. A 102, 6205 (1998)

${ }^{35}$ P. Możejko and L. Sanche, Radiat. Environ. Biophys. 42, 201 (2003).

${ }^{36}$ F. A. Gianturco and R. R. Lucchese, J. Chem. Phys. 120, 7446 (2004).

${ }^{37}$ A. Grandi, F. A. Gianturco, and N. Sanna, Phys. Rev. Lett. 93, 048103 (2004).

${ }^{38}$ S. Tonzani and C. H. Greene, J. Chem. Phys. 124, 054312 (2006).

${ }^{39}$ P. D. Burrow, J. Chem. Phys. 122, 087105 (2005).

${ }^{40}$ C. Winstead and V. McKoy, J. Chem. Phys. 125, 174304 (2006).

${ }^{41}$ C. Winstead and V. McKoy, J. Chem. Phys. 125, 244302 (2006).

${ }^{42}$ C. Winstead and V. McKoy, J. Chem. Phys. 125, 074302 (2006).

${ }^{43}$ K. Takatsuka and V. McKoy, Phys. Rev. A 24, 2473 (1981).

${ }^{44}$ K. Takatsuka and V. McKoy, Phys. Rev. A 30, 1734 (1984).

${ }^{45}$ C. Winstead and V. McKoy, Adv. At., Mol., Opt. Phys. 36, 183 (1996).

${ }^{46}$ C. Winstead and V. McKoy, Comput. Phys. Commun. 128, 386 (2000).

${ }^{47}$ M. W. Schmidt, K. K. Baldridge, J. A. Boatz et al., J. Comput. Chem. 14, 1347 (1993).

${ }^{48}$ M. J. Frisch, G. W. Trucks, H. B. Schlegel et al., Gaussian 94, Revision D.4, Gaussian, Inc., Pittsburgh, PA, 1995.

${ }^{49}$ N. Foloppe and A. D. MacKerell, Jr., Biophys. J. 76, 3206 (1999).

${ }^{50}$ G. Schaftenaar and J. H. Noordik, J. Comput.-Aided Mol. Des. 14, 123 (2000).

${ }^{51}$ T. H. Dunning, Jr., J. Chem. Phys. 55, 716 (1971).

${ }^{52}$ T. H. Dunning, Jr. and P. J. Hay, in Methods of Electronic Structure Theory, edited by H. F. Schaefer III (Plenum, New York, 1977), p. 1.

${ }^{53}$ C. W. Bauschlicher, J. Chem. Phys. 72, 880 (1980).

${ }^{54}$ C. Winstead and V. McKoy, Phys. Rev. A 57, 3589 (1998).

${ }^{55}$ T. N. Rescigno and B. I. Schneider, Phys. Rev. A 45, 2894 (1992), and references therein.

${ }^{56}$ C. Winstead and V. McKoy, Phys. Rev. A 41, 49 (1990).

${ }^{57}$ S. Tonzani and C. H. Greene, J. Chem. Phys. 122, 014111 (2005).

${ }^{58}$ C. Winstead and V. McKoy, Phys. Rev. Lett. 98, 113201 (2007). 